\title{
Antithrombotic treatment in COVID-19 - from theory to practical approach
}

\author{
Ionela-Larisa Miftode ${ }^{1,2}$, Angela Moloce ${ }^{3}$, Radu-Stefan Miftode ${ }^{3,4}$, Viviana Onofrei ${ }^{3,4}$ \\ ${ }^{1}$ Department of Infectious Diseases (Internal Medicine II), \\ "Gr.T. Popa" University of Medicine and Pharmacy, lasi, Romania \\ 2"Sf. Parascheva" Clinical Hospital of Infectious Diseases, Iasi, Romania \\ 3"Sf. Spiridon" Emergency Clinical Hospital, Iasi, Romania \\ ${ }^{4}$ Department of Cardiology (Internal Medicine I), "Gr.T. Popa" University of Medicine and \\ Pharmacy, Iasi, Romania
}

\begin{abstract}
Although initially considered a strictly respiratory pathology, the novel coronavirus disease-19 (COVID-19) has emerged as a significant prothrombotic trigger, inducing hypercoagulable status and increased risk of thrombotic events. This is due to a plethora of mechanisms, either from inflammation-induced endothelial dysfunction, overexpression of procoagulant molecules doubled by down-regulation of physiological antithrombotic pathways, or from an exagerated response to otherwise normal procoagulant stimuli. This complex association of factors define the concept of immunothrombosis, which can be influenced by several antithrombotic medications. Despite the lack of an „universal" guideline, the general consensus is to recommend antithrombotic treatment in COVID-19 patients, but its administration should take into account the patient's clinical status, comorbidities or the other previous indications for antithrombotic treatment. This precaution is due to the multiple drug interactions with antivirals or other molecules used in COVID-19. Concerning anticoagulant treatment, heparins are the optimal choice, compared to antivitamins $\mathrm{K}$ and direct oral anticoagulants (DOACs), because they exhibit the most protective effects doubled by the least interactions with other substances. Hospitalized patients should receive prophylactic doses of anticoagulation, but not for the prevention of arterial thrombosis, unless they have a previous indication such as atrial fibrillation or prosthetic valve. It is generally recommended that patients on chronic anticoagulant or antiplatelet therapy for other conditions will continue their prescribed medication, here including special categories such as pregnant women. However, non-hospitalized patients with mild forms of the disease should not be initiated anticoagulant and antiplatelet therapy unless they have other indication. Continuation of prophylaxis after discharge is a matter of debate, the existing data suggesting it may be considered in those patients at high risk for venous thromboembolism (VTE) and/or who had a moderate-severe form of the disease, always assessing the bleeding risk. Further data from extensive studies are required in order to standardize the antithrombotic approach in COVID-19 patients.
\end{abstract}

Keywords: COVID-19, coagulopathy, antithrombotic agents

\section{INTRODUCTION}

COVID-19 is pathophysiologically characterized by endothelial injury, inflammation and micro- and macrovascular thrombosis, a substrate that justifies the use of antithrombotic treatment. The evolution to arterial and venous thrombotic complications is a common manifestation for patients with severe forms of the disease. 
Regarding daily clinical practice and COVID-19, the following aspects should be underlined: the high prevalence of in situ microvascular localization, that are difficult to predict and to identify, caused via various mechanisms; the generally positive outcomes of an early routine thromboprophylaxis in hospitalized patients, which furtherly raises the need to stratify the risk of thrombosis; the difficulty of quantifying the thrombotic risk in a heterogeneous population, doubled by a chalenging healthcare access. Moreover, in those patients the prognosis is assessed differently depending on studies, consequently the thromboprophylaxis regimens being variably reported in terms of efficiency, safety, and drug types.

For these reasons, antithrombotic treatment recommended in rapidly developing guidelines based on non-randomized studies - remains marked by areas of uncertainty and unanswered questions (1).

\section{PROPOSED MECHANISMS FOR COAGULATION ABNORMALITIES IN COVID-19}

Although the mechanisms that activate the coagulation cascade are not fully elucidated, there is evidence to support some pathogenic links (2).

The first mechanism involves the direct binding of the virus to the host cell membrane and the intervention of the renin-angiotensin system (3). Fusion is accomplished by binding the viral spike protein to the membrane receptors for angiotensin converting enzyme 2 (ACE-2). ACE-2 is a counterregulatory carboxypeptidase of ACE, with an essential role in cardiovascular homeostasis by reducing the production of angiotensin II (Ang II). The consequence of fusion is the „down-regulation” phenomenon concerning the expression of ACE-2 at the cell surface and the subsequent accumulation of Ang II. This aspect increases the expression of tissue factor (TF), which is a key element in triggering the procoagulant response by thrombin formation (3). Thrombin plays a central role because it activates platelets via the protease-activated receptor (PAR-1) and plasma coagulation factors V and VIII. In a similar way, the reduction of ACE-2 activity induces a decrease in Ang 1-7 production which physiologically inhibits platelet adhesion and aggregation by stimulating nitric oxide release in platelets.

The second important mechanism is the response to inflammation (4). Excessive production of proinflammatory cytokines caused by SARS-CoV-2 infection triggers hyperactivation of the immune response, a phenomenon known as the cytokine storm. There is a direct link between inflammatory status and altered coagulation, called immunothrombosis. Proinflammatory cytokines stimulate the local procoagulant response by inducing TF expression in monocytes and „up-regulating” its expression in endothelial cells. Associated platelet activation by von Willebrand factor via specific GPlba glycoprotein receptors occurs. Mobilization of coagulation factors and activated platelets in anatomical sites invaded by SARS-CoV-2 appears to amplify the proinflammatory cytokine production, thrombin synthesis, and in situ thrombus formation. An essential role is also played by neutrophils, which release extracellular particles of neutrophils, generating inflammation, additional thrombin production, exaggerated immune response and thus thrombosis and organ failure (2). All these aspects are additional to a deficient synthesis, consumption and excessive degradation of antithrombin III (2).

Vascular endothelium is another target of SARSCoV-2 infection. Impaired integrity of the vascular endothelium generates platelet activation and thrombus formation. It is well established the diabetes involment in endothelial dysfunction; hence, dabetic patients are much more vulnerable to thrombotic events (5).It is also stated that, in some patients, the endothelium also represents an initial site for intravascular disseminated coagulation (2).

Thrombocytopenia, reported as a common manifestation in patients with severe infection, is caused by extensive damage to bronchoalveolar tissue and endothelial cells and is accompanied by massive platelet recruitment and consumption following intense activation (6). For the clinician, thrombocytopenia does not represent an indication for stopping antithrombotic treatment, but only a closer followup after choosing the right type of medication and its adequate posology.

Alteration of the natural fibrinolytic response completes the drama of coagulation in COVID-19, as both processes excessively coexist. Hyperfibrinolysis is considered a mark of severe evolution. Cui et al. reports that in patients with severe pneumonia and venous thromboembolism (VTE), D-dimers, as fibrin degradation products, are intensely elevated and are accurate biomarkers for thrombosis (7).

\section{PATHOPHYSIOLOGICAL ARGUMENTS FOR ANTITHROMBOTIC TREATMENT}

The immunothrombosis process can be influenced in both components of anticoagulant treatment, by reducing the thrombotic load and the hyperreactivity of the coagulation process as well as by the anti-inflammatory effect. Heparins block selectin-P, an adhesion molecule with an essential role in the leukocytes recruitment at the site of inflammation, selectin $\mathrm{E}$ and the intercellular adhesion molecule (ICAM-1), with a role in promoting inflammation and cell signaling. Heparins also 
block the interaction between platelets and neutrophils, inhibit the production of extracellular neutrophilic particles and, most importantly, reduce the release of IL-1 $\beta$ and IL- 6 for which there is evidence of hypercoagulation and thrombi, including in patients with severe COVID-19 (8). The beneficial effects continue through the heparins inhibition of the proteolysis process (via cathepsins, factor Xa, furin and trypsin). Proteolysis is essential for cell infection and causes cleavage of S1-S2 subunits of spike proteins. In particular, activated factor $\mathrm{X}$ facilitates the activation of SARS-CoV-2 penetration into cells (9).

Unlike anticoagulant treatment for which there is clinical and prognostic evidence regarding its favorable effect, for antiplatelet treatment the administration is based only on pathophysiological arguments from experimental models of viral infection. Inflammation and endothelial dysfunction may represent a trigger for platelet adhesion and aggregation, initiating the thromboinflammatory process. It is well known that uncontrolled platelet activation causes extensive inflammation. Another category of evidence came from experimental studies demonstrating the beneficial effects of specific antiplatelet medication (aspirin, eptifibatide, clopidogrel, protease-activated receptor 4 antagonists) in viral infections, which protect the lungs from severe injury, limit virus spread and immune activation. However, the degree of platelet activation in COVID-19 is currently unknown and therefore the prophylactic role of antiplatelets (either single or dual medication) on the viral load and clinical course of the disease is still to be elucidated (10). The currently available clinical data emphasize on the idea that the optimal medication is not necessarily the antiviral one, but the pathogenic, host-related treatment, in order to modulate thromboinflammation. From this point of view, the use of antiplatelet medication together with steroidal antiinflammatory and immunomodulatory drugs seems to be a rational therapeutic target. A practical example of the benefit of antiplatelet medication is the effect of hydroxychloroquine, initially used empirically, but for which antiplatelet properties have been demonstrated, through the possible interaction with the arachidonic acid pathway and the fibrinogen reduction (11).

\section{ANTITHROMBOTIC MEDICATION AVAILABLE FOR COVID-19}

There is currently no solid information on the protective effect of anticoagulants and antiplatelet agents, thereby their administration should take into account the clinical status of the patient, the comorbidities and other previous indications for an- tithrombotic medication, because there are multiple drug interactions with antivirals or other drugs used in COVID-19. Heparins are the optimal choice, compared to antivitamins $\mathrm{K}$ and direct oral anticoagulants (DOACs), because they have the most protective effects and the least interactions with other substances (Table 1).

\section{RANDOMIZED TRIALS IN PATIENTS WITH COVID-19}

The interest in antithrombotic treatment is justified by the increased incidence of thrombotic events, in particular venous thrombembolism (VTE). On the other hand, the septic status in COVID-19 induces a hypercoagulability status and in situ microthrombosis. The current international experience has led to the argument that there is no „onesize-fits-all" solution, because there are many variables that condition the treatment regimen in the patient with COVID-19. Randomized trials are the most accurate way to evaluate the effectiveness of a medication that is already widely used but requires strict individualization and adaptation.

One year after the onset of the pandemic, there are numerous ongoing randomized trials (RCTs) evaluating various therapeutic regimens with antiplatelet agents, various types of anticoagulants including DOACs, fibrinolytics, or a combination thereof. The intensity of therapeutic regimens is variable, depending on the estimated risk of thrombotic events, proportional to the severity of the infection. The disadvantages of the trials are the openlabel design and the fact that they exclude patients at high risk of bleeding, with severe renal dysfunction, pregnant women or obese people. More than half of the trials evaluating regimens, other than standard anticoagulation regimens, exclude patients with creatinine clearance $<15 \mathrm{ml} / \mathrm{min}$. The objectives pursued vary depending on the population studied and include all-cause mortality, arterial and venous thromboembolic risk, need for hospitalization/re-admission, hospitalizations for cardiovascular/pulmonary events, need for respiratory support, or composite targets. The most common safety goal is the risk of bleeding.

\section{RECOMMENDATIONS FOR ANTITHROMBOTIC THERAPY}

Although there are several available recommendations and guidelines of national societies, this paper will summarize the antithrombotic treatment recommendations launched by the National Institutes of Health (NIH). The recommendations are included in the treatment guideline for COVID-19, available on the official NIH website, which is regularly updated (12). 
TABLE 1. Antithrombotic medication used in COVID-19 - adapted after (8)

\begin{tabular}{|c|c|c|c|}
\hline Antithrombotic agent & Protective effect & Clinical situation & Interactions with other drugs \\
\hline \multicolumn{4}{|l|}{ Antiplatelets } \\
\hline Aspirine & Platelet activity reduction & IHD & - \\
\hline Clopidogrel & $\begin{array}{l}\text { MMPs } \\
\text { monocytes, neutrophils }\end{array}$ & $\begin{array}{l}\text { IHD } \\
\text { Stroke }\end{array}$ & $\begin{array}{l}\text { No administration / dose reduction with } \\
\text { Lopinavir / Ritonavir (CYP3A4) }\end{array}$ \\
\hline Ticagrelor & NF-kB effect attenuation & Stroke & - \\
\hline Prasugrel & $\begin{array}{l}\text { inflammatory response } \\
\text { viral spread }\end{array}$ & $\begin{array}{l}\text { PAD } \\
\text { ACS }\end{array}$ & $\begin{array}{l}\text { No administration / dose reduction with } \\
\text { Lopinavir / Ritonavir (CYP3A4) }\end{array}$ \\
\hline Cilostazol & $?$ & $\begin{array}{l}\text { ACS } \\
\text { PAD }\end{array}$ & $\begin{array}{l}\text { Caution with Lopinavir / Ritonavir (CYP3A4) } \\
\text { Maximum dose } 50 \mathrm{mg} \text { with Lopinavir / } \\
\text { Ritonavir (CYP3A4) }\end{array}$ \\
\hline \multicolumn{4}{|l|}{ Anticoagulants } \\
\hline Heparins & \begin{tabular}{|l|} 
Structural changes: S1 binding \\
Virus attachment: competition with HSBG \\
Virus-cell fusion: S1-S2 cleavage \\
Anti-inflammatory mechanism: IL-6, \\
NF-kB \\
VTE prophylaxis: microvascular \\
thrombosis
\end{tabular} & \begin{tabular}{|l|} 
AF \\
VTE \\
ACS, Stroke \\
Mechanical \\
cardiac \\
prostheses
\end{tabular} & \\
\hline Antivitamin $\mathrm{K}$ & $\begin{array}{l}\text { VTE prophylaxis: microvascular } \\
\text { thrombosis }\end{array}$ & $\begin{array}{l}\text { AF, VTE, } \\
\text { ACS, Stroke } \\
\text { Mechanical } \\
\text { cardiac } \\
\text { prostheses }\end{array}$ & $\begin{array}{l}\text { Dose increase: Lopinavir / Ritonavir, } \\
\text { Ribavirin } \\
\text { Dose reduction: Interferon, Azithromycin, } \\
\text { Methylprednisolone }\end{array}$ \\
\hline Apixaban & \multirow[t]{4}{*}{$\begin{array}{l}\text { VTE prophylaxis: microvascular } \\
\text { thrombosis }\end{array}$} & \multirow{4}{*}{ AF, VTE, ACS } & $\begin{array}{l}50 \% \text { dose (avoidable if the initial dose is } \\
2.5 \mathrm{mg} \times 2 \text { / day) with Lopinavir / Ritonavir } \\
\text { (CYP3A4, P-gp) }\end{array}$ \\
\hline Rivaroxaban & & & $\begin{array}{l}\text { No administration withLopinavir / Ritonavir } \\
\text { (CYP3A4, P-gp) }\end{array}$ \\
\hline Dabigatran & & & - \\
\hline Edoxaban & & & $\begin{array}{l}\text { No administration with Lopinavir / Ritonavir } \\
\text { (CYP3A4, P-gp) } \\
\text { VTE: } 30 \mathrm{mg} / \text { day with Azithromycin (P-gp) }\end{array}$ \\
\hline
\end{tabular}

IHD - ischemic heart disease; $A C S$ - acute coronary syndrome; MMP- matrix-metallo proteinases; $P A D$ - peripheral artery disease; $A F-$ atrial fibrillation; VTE - venous thromboembolism; HSPG - heparan sulfate proteoglycan; CYP3A4 - cytochrome P450 3A4; P-gp - 1P glycopreotein

According to the guideline, there are currently insufficient data to support or combat the assessment of coagulation parameters, such as D-dimers, prothrombin time, platelets or fibrinogen in hospitalized patients receiving antithrombotic treatment. The same parameters are not required to be assessed in ambulatory. The guideline also does not support routine screening for deep vein thrombosis in patients who do not have a clinical aspect suggestive of VTE. Instead, VTE should be evaluated in any hospitalized patient with rapidly deteriorating pulmonary, cardiovascular, or neurological function.

Patients on chronic anticoagulant or antiplatelet therapy for other conditions will continue their prescribed medication, here including special categories such as pregnant women or nursing mothers. In these cases, UFH, LMWH and antivitamins $\mathrm{K}$ (AVK) can be safely used, but not DOACs.

\section{VTE SCREENING AND PROPHYLAXIS}

Non-hospitalized patients should not be initiated anticoagulant and antiplatelet therapy unless they have other indication or participating in clinical trials.

Hospitalized patients should receive prophylactic doses of anticoagulation, but not for the prevention of arterial thrombosis, unless they have a previous indication such as atrial fibrillation. There are is no clear data on whether or not to support higher doses of anticoagulants for VTE prophylaxis. However, if patients have a history of VTE or if there is a high suspicion for a first episode and the imaging confirmation is not possible, curative doses of anticoagulant will be initiated. Also, antithrombotic treatment should be applied according to non-COVID protocols in critical patients who require extracorporeal oxygenation, dialysis or have catheter thrombosis. Prophylactic doses of anticoagulation are recommended in pregnant women hospitalized for severe forms of COVID-19.

Continuation of prophylaxis after discharge can only be considered in those patients at high risk for VTE and low bleeding risk, including pregnant or breastfeeding/nursing mothers. The FDA has approved the use of rivaroxaban $10 \mathrm{mg}$ for 31-39 days, 
TABLE 2. Prophylactic anticoagulation regimens according to WHO - adapted after (1)

\begin{tabular}{|c|c|c|}
\hline Anticoagulant type & Standard dose & Dose adjusted \\
\hline Unfractionated heparin & 5,000 UI s.c. $\times 2 /$ day & \\
\hline Enoxaparin & $\begin{array}{l}40 \text { mg/day s.c., including post } \\
\text { discharge for } 45 \text { days }\end{array}$ & $\begin{array}{l}\mathrm{CrCl}<30 \mathrm{ml} / \mathrm{min}-20 \mathrm{mg} / \text { day s.c. } \\
\mathrm{BMI} 30-40 \mathrm{k} / \mathrm{m}^{2}-40-60 \mathrm{mg} / \text { day s.c. } \\
\mathrm{BMI}>40 \mathrm{~kg} / \mathrm{m}^{2}-40 \mathrm{mg} \times 2 / \text { day s.c. } \\
\mathrm{BMl}>50 \mathrm{~kg} / \mathrm{m}^{2}-60 \mathrm{mg} \times 2 / \text { day s.c. }\end{array}$ \\
\hline Fondaparin & $2.5 \mathrm{mg} /$ day & \\
\hline Rivaroxaban & $\begin{array}{l}\text { Post discharge } 10 \mathrm{mg} / \text { day for up to } \\
45 \text { days }\end{array}$ & \\
\hline
\end{tabular}

$\mathrm{CrCl}$-creatinine clearance; $\mathrm{BMI}-$ body mass index

and the risk assessment requires the calculation of IMPROVE and modified IMPROVE risk scores. Increased risk is defined as an IMPROVE score $\geq 4$ or modified IMPROVE score $\geq 2$ with D-dimers twice the upper normal limit (13-15). In parallel, it is necessary to quantify the risk of bleeding and the possibility of patients follow-up.

Regarding therapeutic doses of anticoagulants for VTE prevention, there are limited data from prospective studies. There are also numerous guidelines developed by companies and experts that have reached a consensus concerning the prophylactic doses. There is no consensus on the use of intermediate doses in critical patients $(16,17)$.

The type of antithrombotic medication must be selected taking into acocunt the drug interactions and the possibility of monitoring. In critically-ill, hospitalized patients, UFH or LMWH are preferred because they can be more easily manipulated and have fewer interactions. Patients who have another indication for chronic anticoagulation and are already under treatment with VKA may be candidates for DOACs if there are no contraindications (mechanical valve prostheses, mitral stenosis, ventricular assist devices, phospholipid syndrome, pregnancy).

In pregnant women, it is not known whether COVID-19 infection further increases the risk of thromboembolism $(18,19)$. The Society of Maternal and Fetal Medicine recommends prophylactic doses of heparin or preferably HGGM, in critically ill or mechanically ventilated patients, unless there are other contraindications. Similarly, the risk of bleeding should be assessed very carefully (20). The use of risk scores and D-dimers assessment is not very accurate in estimating the probability of $\operatorname{VTE}(21,22)$.
Prophylactic anticoagulation regimens can be applied by any of the specialists treating patients with COVID-19 (Table 2). Therapeutic regimens will be applied individually, following a discussion with the cardiologist.

A special mention is required concerning the safe administration of antiplatelet medication, related to the COVID-19 infection potential to cause thrombocytopenia. If platelets are between 25,000 and $50,000 / \mathrm{mm}^{3}$, a single antiplatelet agent will be used, while a level $<25,000 / \mathrm{mm}^{3}$ requires the stoping of antiplatelet medication (8).

A simplified anticoagulation management algorithm would be the following (8):

- in mild forms of COVID-19, early mobilization or pharmacological thromboprophylaxis in patients at high risk of VTE is encouraged;

- in moderate and severe forms of COVID-19 it is absolutely necessary to assess the risk of VTE, but also the contraindications for anticoagulants/antiplatelets administration. In this situation, prophylactic anticoagulation or intermittent pneumatic compression is mandatory in patients without other contraindications $(1,8)$. In situations that evolve with clinical or hemodynamic degradation or in the cases with a high possibility for drug interactions, UFH or LMWH will be chosen as appropriate.

\section{CONCLUSIONS}

The dynamic evolution of COVID-19 pandemic and the need to promptly manage the risk of thrombotic events in patients infected with SARS-CoV-2 requires well-informed and individualized therapeutical approach, until ongoing clinical trials will provide the much-needed validated data.

Conflict of interest: none declared Financial support: none declared

\section{REFERENCES}

1. Ramacciotti E, Macedo AS, Biagioni RB, et al. Evidence-Based Practical Guidance for the Antithrombotic Management in Patients With Coronavirus Disease (COVID-19) in 2020. Clin Appl Thromb Hemost. 2020;26:1-8.

2. Sathler PC. Hemostatic abnormalities in COVID-19: A guided review. An Acad Bras Ciênc. 2020;92(4):e20200834.

3. Guo J, Huang Z, Lin L, Lv J. Coronavirus Disease 2019 (COVID-19) and Cardiovascular Disease: A Viewpointon the Potential Influence of 
Angiotensin-Converting Enzyme Inhibitors/Angiotensin Receptor Blockers on Onset and Severity of Severe Acute Respiratory Syndrome Coronavirus 2 Infection. J Am Heart Assoc. 2020;9(7):e016219.

4. Vaninov N. In the eye of the COVID-19 cytokine storm. Nat Rev Immunol. 2020;20(5):277.

5. Miftode E, Miftode L, Coman I, et al. Diabetes Mellitus - A Risk Factor for Unfavourable Outcome in COVID-19 Patients - The Experience of an Infectious Diseases Regional Hospital. Healthcare. 2021;9:788.

6. Lippi G, Plebani M, Henry BM. Thrombocytopenia is associated with severe coronavirus disease 2019 (COVID-19) infections: A metaanalysis. Clin Chim Acta. 2020;506:145-148.

7. Cui S, Chen S, Li X, Liu S, Wang F. Prevalence of venous thromboembolism in patients with severe novel coronavirus pneumonia. J Thromb Haemost. 2020;18(6):1421-1424.

8. Godino C, Scotti A, Maugeri N, et al. Antithrombotic therapy in patients with COVID-19? Rationale and Evidence. Int J Cardiol. 2021;324:261-266

9. Zhou F, Yu T, Du R, et al. Clinical course and risk factors for mortality of adult inpatients with COVID-19 in Wuhan, China: a retrospective cohort study. Lancet. 2020;395:1054-1062.

10. Mycroft-West CJ, Su D, Elli S, et al. The 2019 coronavirus (SARSCoV-2) surface protein (Spike) S1 receptor binding domain undergoes conformational change upon heparin binding. BioRxiv 2020.

11. Achuthan S, Ahluwalia J, Shafiq N, et al. Hydroxychloroquine's efficacy as an antiplatelet agent study in healthy volunteers: A proof of concept study. J Cardiovasc Pharmacol Ther. 2015;20:174-180.

12. NIH COVID-19 Treatment Guidelines. Antithrombotic Therapy in Patients With COVID-19. Available at: https://www. covid19treatmentguidelines.nih.gov/, april 2021.

13. Roberts LN, Whyte MB, Georgiou L, et al. Postdischarge venous thromboembolism following hospital admission with COVID-19. Blood. 2020;136(11):1347-1350.

14. Patell R, Bogue $T$, Koshy $A$, et al. Postdischarge thrombosis and hemorrhage in patients with COVID-19. Blood. 2020; 136(11):1342-1346.
15. Spyropoulos AC, Lipardi C, Xu J, et al. Modified IMPROVE VTE risk score and elevated D-dimer identify a high venous thromboembolism risk in acutely ill medical population for extended thromboprophylaxis. TH Open. 2020;4(1):e59-e65.

16. Barnes GD, Burnett A, Allen A, et al. Thromboembolism and anticoagulant therapy during the COVID-19 pandemic: interim clinical guidance from the anticoagulation forum. J Thromb Thrombolysis. 2020;50(1):72.

17. Royal College of Physicians. Clinical guide for the prevention, detection and management of thromboembolic disease in patients with COVID-19. Available at: https://icmanaesthesiacovid-19.org/ clinical-guide-prevention-detection-and-management-of-vte-inpatients-with-covid-19, 2020.

18. Knight M, Bunch $\mathrm{K}$, Vousden $\mathrm{N}$, et al. Characteristics and outcomes of pregnant women admitted to hospital with confirmed SARS-CoV-2 infection in UK: national population based cohort study. BMJ. 2020;369:m2107.

19. Delahoy MJ, Whitaker M, O'Halloran A, et al. Characteristics and maternal and birth outcomes of hospitalized pregnant women with laboratory-confirmed COVID-19 - COVID-NET, 13 states. MMWR Morb Mortal Wkly Rep 2020;69(38):1347-1354.

20. Society for Maternal Fetal Medicine. Management considerations for pregnant patients with COVID-19. Available at: https://s3.amazonaws. com/cdn.smfm.org/media/2336/SMFM, 2020.

21. Miftode E, Luca C, Manciuc C, et al. Covid-19: A course through stormy waters. Med Surg J Rev Med Chir Soc Med Nat (lasi). 2020; 124:351-362.

22. Hu W, Wang Y, Li J, et al. The predictive value of D-dimer test for venous thromboembolism during puerperium: a prospective cohort study. Clin Appl Thromb Hemost. 2020;26:1076029620901786. 